\title{
Tras las huellas del aprendizaje: un proceso reflexivo sobre la infancia aprendiente ${ }^{*}$
}

\section{In the footsteps of learning: A reflection on childhood learning}

\author{
Ada Marcela González Riveros ${ }^{1}$, Natalia Andrea Rodríguez Salamanca ${ }^{(0}$
}

Para citar este artículo: González, A. M., Rodríguez, N. A. (2019). Tras las huellas del aprendizaje: un proceso reflexivo sobre la infancia aprendiente. Infancias Imágenes, 18(2). [278-284]

\section{Resumen}

El presente texto tiene como propósito reflexionar sobre el aprendizaje en la infancia y su relación con los instancia, se abordan transformaciones que ha tenido el aprendizaje como objeto de estudio, desde la perspectiva experimental hasta los postulados de la neurociencia. Acto seguido se entra en diálogo con los principios de la autoorganización y sus implicaciones en el aprendizaje de la infancia; y, por último, se considera el principio de enacción propuesto por Varela para reconfigurar el acto de aprender desde la infancia, que juega y explora para desarrollar sus procesos cognitivos, acercándose a la comprensión del concepto de infancia aprendiente. En conclusión, el artículo busca ampliar la mirada sobre el aprendizaje con la intención de retornar a la sensibilidad de la infancia que posee la posibilidad de construir procesos de comprensión y resignificación de realidad mediante su accionar en el mundo.

Palabras clave: aprendizaje, infancia aprendiente, enacción, juego.

\begin{abstract}
This study reflects upon learning during childhood and its relationship with vital processes and experiences of a child. First, the transformations of learning as the subject of study both from an experimental as well as from a neuroscientific perspective are addressed. Second, a discussion on self-organization principles and their implications for childhood learning is conducted. Last, the principle of enacting proposed by Varela is considered to reconfigure the act of learning since childhood when playing and exploring are ways of developing cognitive processes, bringing us closer to the understanding of the concept of a childhood learner. In conclusion, this study seeks to widen the view of learning with the intention of focusing on the sensitivity of childhood with its potential of building comprehension mechanisms and redefining reality through worldly actions.
\end{abstract}

Keywords: learning, learner childhood, enacting, game.

* Artículo de reflexión resultado de proceso de formación del rizoma doctoral.

1 Doctoranda en Educación, Universidad de La Salle Costa Rica. Magíster en Educación, Pontificia Universidad Javeriana. Máster en Neuropsicología y Educación, Universidad Internacional de La Rioja, España. Docente tiempo completo, Corporación Universitaria Minuto de Dios. Correo electrónico: amgonzalez@uniminuto.edu

2 Doctoranda en Educación, Universidad de La Salle Costa Rica. Magíster en Docencia, Universidad de La Salle Colombia. Licenciada en Ciencias Sociales, Universidad Pedagógica y Tecnológica de Colombia. Docente tiempo completo, Corporación Universitaria Minuto de Dios. Correo electrónico: natalia.rodriguez@uniminuto.edu 
"Los niños viven sus vidas a través de las infancias construidas para ellos por las interpretaciones que las personas adultas tienen de la infancia y de lo que son y deberían ser los niños"

Berry Mayall (1996, p. 1)

\section{Introducción}

La especia humana ha evolucionado a partir de un proceso biológico y cultural que emerge de la esencia de la vida y que configura las relaciones que se tejen en interdependencia con el ambiente. Bajo este panorama, la infancia, como parte de la humanidad, guarda estas características y construye su identidad a partir de la semiótica lingüística o corporal que comunican la particularidad de lo que sienten al experimentar las construcciones dadas entre el sujeto-sujeto y sujeto-entorno. No obstante, esta singularidad en ocasiones se ve opacada por las demandas excesivas que surgen en los entornos familiares o educativos y llevan a los niños a vivir un conflicto interno entre las capacidades innatas, los deseos de aprender y las expectativas que tienen los mismos sobre su potencial de aprendizaje.

Esta situación no es nueva y mucho menos ajena a los estudios que se han realizado desde disciplinas como la neurociencia y la pedagogía, pues cada una desde su naturaleza unen esfuerzos para dar explicaciones al fenómeno del aprendizaje desde una perspectiva holística. Al respecto, Pérez y Álvarez (2014), en su estudio sobre la motivación de los niños de primaria, expresan que hay factores asociados a los contextos que inciden en el desarrollo socioemocional y en el desempeño escolar; pero dejan entrever que la motivación por aprender sigue latente pese a las situaciones adversas que se presentan en el acto de aprender. Este resultado demuestra que los niños no sienten desmotivación por saber, descubrir o crear nuevas formas de representar el mundo, sino que siempre están en disposición para accionar y construir nuevos conocimientos.

Por otra parte, Martínez, Pérez y Sierra (2014) indagan sobre la opinión que tiene el profesorado consultado sobre la influencia de los hábitos educativos englobados en el currículo de Educación Infantil, así como en la adquisición de aprendizajes. Los resultados demuestran que los hábitos morales, conductuales y sociales son importantes en el desarrollo de capacidades cognitivas, afectivas y de interacción social; por lo cual, las perspectivas socioconstructivistas favorecen el acto de aprender y en esa línea los profesores deben dar relevancia a actividades diversificadas que contribuyan a una formación holística.

En este sentido, se reconoce que las prácticas que homogenizan la experiencia de aprendizaje de los niños no contribuyen al reconocimiento de las múltiples maneras de aprender como lo expresa Assmann, quien hace un llamado sobre esta circunstancia al afirmar que: "se aprende no sólo con el cerebro ni sólo en la escuela. Se aprende durante toda la vida y mediante todas las formas de vivir" (2002, p. 11). Este pensamiento aporta de manera significativa a la comprensión del niño como sujeto aprendiente, que se encuentra en proceso activo y permanente de aprendizaje a partir de las experiencias, que desde el pensamiento de Dewey se refieren "al intercambio de un ser vivo con su medio ambiente físico y social y no solamente un asunto de conocimiento" (Ruiz, 2013, p. 107).

En consecuencia, este texto pretende abordar algunas huellas que contribuyen a la comprensión de los procesos de aprendizaje y el reconocimiento que tiene la infancia dentro de estos. Para su desarrollo se hace una recopilación documental como parte de la construcción de un estado del arte frente a las categorías aprendizaje e infancia, con el fin de reconocer el concepto de infancia aprendiente propuesto por Assmann (2002) en las dinámicas propias de esta población.

\section{El aprendizaje como objeto de estudio} La tradición objetivista que permea la comprensión de los actos de aprendizaje tiene su origen en el surgimiento de enfoques basados en la disociación del cuerpo y de la mente. Así es como en la antigüedad los planteamientos de Platón, y en la modernidad los de Descartes, ya evidenciaban una tendencia a relacionar el conocimiento como un producto racional del acto de aprender. Con el tiempo aumentó la necesidad de explicar la naturaleza del conocer y fue en ese contexto en el que se aguzó el interés por el desarrollo de teorías en el siglo XX, hasta el punto de identificar y enfocar 
las relaciones que se establecen entre el medio y los individuos a partir de la investigación en torno a los comportamientos humanos.

De esta forma, aprender se asocia con el cambio dado ante los estímulos que surgen en la interacción con los factores controlados del ambiente, y en esta perspectiva experimental el individuo queda ajeno al tratamiento y las variables que median su proceso de transformación interna. A partir de esta idea, Skinner (1953) propone el condicionamiento operante o instrumental apoyado en que el tiempo posibilita la práctica de una conducta observable; más adelante Bandura, dando paso a un cambio de perspectiva del conductismo, "agrega las características sociales del individuo o la capacidad del sujeto para sacar partido de la experiencia para prever o dirigir su acción" (Amigues, 2001, citado por Sánchez y Andrade, 2014, p. 45). Por ello, el aprendizaje en esta lógica emerge del moldeamiento social y el reforzamiento constante.

Estas ideas se retoman para el desarrollo de una enseñanza basada en instrucciones y tareas que involucran un docente, agente o recurso poseedor de un conocimiento que debe ser replicado por infantes en diferentes contextos. Asimismo, ubican a la observación como elemento central para lograr éxito en la adquisición de una conducta/aprendizaje regulado por el medio. Al reflexionar sobre ello se logra evidenciar una lógica de aprendizaje predictivo y controlado por variables externas que configuran patrones de comportamiento que universalizan las respuestas y a la infancia misma. Este elemento se asemeja a las concepciones ambientalistas de infancia que devienen del siglo XVIII, bajo la influencia de la obra de John Locke (1763) quien, en la metáfora de la tabula rasa, cree que los niños se constituyen por las condiciones del medio, las cuales permiten la adquisición de conocimientos que no tienen por la fragilidad que les es propia (Alzate, 2003). Este aspecto no es ajeno a la evolución del aprendizaje en el plano pedagógico, pues Comenius en su obra Didáctica Magna del siglo XVII ya había destacado el papel de método como forma de instrucción en el acto de enseñar y aprender, así como la necesidad de situar un orden en lo que consideraba un modelo cerrado de educación, el cual privilegia el papel del tiempo cronológico para el paso de un no saber a un saber, de una infancia a la adultez. En este sentido, el tiempo de la experiencia que tiene la infancia es incluido en las actividades que comparte en el contexto materno, donde es orientado por principios de instrucción que precisan su aprendizaje antes de la enseñanza en la escuela.

Desde las ideas del conductismo se da paso a la revolución cognitiva de la década de 1960 como reacción a la manera mecánica con la que se asume el acto de aprender y las implicaciones que esto tiene en la comprensión de la estructuración mental del ser humano. Es así como la psicología de la Gestalt declara que el todo es más que la suma de las partes y en esta lógica se percibe la totalidad de la realidad, al contrario de la visión atomista del aprendizaje que niega el contexto donde coexiste el sujeto y las relaciones que se establecen con este. Por su parte, la psicología cognitiva, bajo influencia de la Gestalt, se interesa por los procesos internos que suceden al aprender y, desde allí, el enfoque del procesamiento de la información se encauza a la memoria como centro de almacenamiento de conocimientos adquiridos, reforzando así prácticas de transmisión centradas en el producto y desplazando la actividad del sujeto aprendiente (De Corte, 2016).

Como avance en el estudio del aprendizaje autores como Piaget, Bruner y Ausubel toman diferentes caminos para abordar el estudio de los procesos estructurales del aprendizaje en interacción con el medio y coinciden en que este involucra mecanismos de reconfiguración interna que se gestan al construir conocimientos en la actividad del sujeto. Desde esta perspectiva los procesos de andamiaje, la motivación, la acción, el conflicto cognitivo y la creatividad en las representaciones mentales construidas desde la experiencia permiten que la exterioridad con la que se ha tratado al sujeto en el acto de aprender se movilice a escenarios enactuados, pero aún cargados del interés por develar la actividad interna del sujeto.

En este sentido, la debilidad e incompletitud con la que se había representado a la infancia en el Medioevo se torna porosa al entrar en una paradoja que configura un campo de estudio que da valor a los niños como objeto de investigación o 
conocimiento científico y a la vez afianza escenarios de reclusión parcial o temporal en escuelas que los despojan de ese valor que es tan inquietante para los científicos. Ante este panorama, la preocupación por el aprendizaje de la infancia se bifurca de acuerdo con la información científica del cómo se gesta este proceso y cómo se aplica en la práctica pedagógica que sucede en los entornos escolares. La configuración de este binomio abona terrenos para un pensamiento absolutista que exalta la verdad y homogeneidad en los contenidos, fines, espacios y actores educativos, quienes en su interés por el aprendizaje han proliferado "recetas de enseñanza" aislándose de la complejidad observada por los estudios que enmarcan la acción, la interacción sociocultural y las herramientas que median los contextos en que los niños experimentan actividades que le son propias.

Pese a lo anterior, el auge de las teorías constructivistas sitúa a la infancia en un escenario activo que se logra articular a finales del siglo XX con las ideas de Vygotsky en los aportes de las teorías socioculturales con el fin de profundizar en las relaciones que se establecen en la interacción del sujeto con la cultura. En este marco, el concepto de zona de desarrollo próximo (ZDP) toma fuerza en las estrategias educativas al otorgar un lugar central a los procesos de participación, negociación y producción de conocimientos colectivos que incluyen al docente como generador de espacios de aprendizaje colaborativos, cooperativos y distributivos, en los cuales el lenguaje se convierte en el fundamento para la expresión del pensamiento (De Corte, 2016).

Este giro en las estructuras de pensamiento de la época impulsa ideas progresistas en la pedagogía que aunadas a los avances de la neurociencia ampliaron el escenario del aprendizaje al resignificar las actividades vitales de la infancia. En este mar$\mathrm{CO}$, se evidencia que la proliferación de neuronas en el cerebro, en especial en las áreas frontales e hipocampo, sucede por la plasticidad cerebral y la modificación que tienen las conexiones neuronales al entrar en contacto con ambientes y experiencias estimulantes como el ejercicio y el entrenamiento. Asimismo, el desarrollo cognitivo entra en coherencia con una adecuada estimulación en todas las dimensiones, por lo que las carencias ambientales y en experiencias producen anomalías funcionales y anatómicas (Portellano, 2008).

Las contribuciones al aprendizaje de la infancia se han materializado en la compresión de la complejidad que tienen los periodos sensibles que se inician desde la época prenatal, pues es en ellos donde se conjuga la calidad del ambiente social, cultural, familiar, nutricional, emocional con los aspectos biológicos que devienen de la organización interna del sujeto. Otro aporte de la neurociencia es la forma de abordar la diversidad presente en los niños que confluye en los contextos educativos, pues los estudios sobre estilos de aprendizaje e inteligencias múltiples han logrado deslocalizar la homogeneidad presente en las teorías expuestas en el siglo XX. Los estilos responden a las preferencias que tienen los individuos para percibir, codificar, procesar y representar la información. En este sentido, influyen en las decisiones que se toman, se relacionan con el ¿cómo sé?, ¿cómo pienso?, ¿Cómo me siento y reacciono? y ¿cómo actuó? (Salas, 2014). Las inteligencias ofrecen una gama de posibilidades de desarrollo basadas en el potencial que tienen los sujetos para establecer puentes entre sus capacidades y de esta manera responder a las demandas de los contextos.

Estos descubrimientos impactan la forma en la que se concibe la infancia en singular al dar paso a la comprensión de las realidades diversas que se presentan en el acto de aprender; además, modifican las relaciones del infante con el lenguaje, la matemática y otras disciplinas, pues evidencian la existencia de zonas del cerebro que en articulación con las experiencias corporales se activan formando un todo que responde de manera asertiva a las actividades cotidianas. En este sentido, la visión reduccionista y fragmentada del aprendizaje se reconfigura al tener una mirada holística que supera las nociones "edaistas" que sitúan en un tiempo y espacio fijo al aprendizaje y en su lugar conjugan al cuerpo, la mente y la emocionalidad como dimensiones de la infancia aprendiente, que entran en interdependencia al proporcionar la libertad para crear, imaginar, comunicar y movilizar estructuras a partir del juego, la literatura, la exploración y otras artes. 
En contraposición a estos planteamientos holísticos, existen otros enfoques de estudio del aprendizaje orientados a explicaciones derivadas del auge de la fase cibernética (cinbernhtich), que contribuyen a la consolidación de teorías en el campo de las ciencias cognitivas. Según Ojeda (2001), la cibernética se define como un arte para pilotear, dirigir y gobernar, postulado que con el tiempo se complementa al intentar construir un prototipo de los sistemas de regulación automática equiparado al de los seres vivos, consolidando una ciencia la cual precisa que la mente y la cognición están determinadas por procesos lógicos. En esta línea, se considera que el cerebro es una máquina deductiva que opera con neuronas, que podrían estar activas o inactivas y que las interconexiones son fundamentales para las operaciones lógicas. Este pensamiento posiciona el enfoque representacional simbólico del aprendizaje y a la inteligencia en términos de capacidad para representar el mundo y solucionar problema planteados (Varela, 1998).

Por otro lado, en esta disciplina también se incluye los sistemas autoorganizativos que permiten "la comprensión de los organismos vivos como sistemas energéticamente abiertos, pero organizativamente cerrados" (Capra, 1996, p. 96). Es así que la comprensión del patrón y su relación con los procesos de configuración en red de los seres vivos se convierte en pieza clave para develar los procesos de aprendizaje. Con el tiempo, los estudios en relación con los procesos de autoorganización evidenciaron que estos sistemas guardan propiedades para establecerse de manera autónoma lejos del equilibrio y que esta característica da paso a la creación de nuevas estructuras que se interconectan de manera no lineal (Capra, 1996). Es decir, para que se geste el aprendizaje se deben dar procesos emergentes que lleven a configurar nuevas estructuras, permeadas por movimientos y transformaciones que permiten un nuevo acoplamiento estructural.

En este orden de ideas, y basados en los aportes que se identificaron, se orienta la mirada hacia lo que se denomina cibernética de segundo orden, definida como el "estudio de los sistemas en los cuales nuestra propia actividad descriptiva es parte de los mismos" (Maturana y Varela, 2003, p. XX). Desde esta perspectiva, el estudio del aprendizaje retorna a su esencia por medio de la investigación de la percepción y organización del ser vivo y con ello se da lugar a las teorías que se inclinan por la comprensión de las conexiones existentes entre las redes de neuronas y los movimientos emergentes que suscitan cambios en la estructura. Dicho de otro modo: "los cerebros operan de forma distribuida a partir de interconexiones masivas, de modo que las conexiones reales entre los conjuntos de neuronas cambian como resultado de la experiencia" (Varela, Thompson y Rosch 1997, p. 111). Por esto, en las experiencias de la infancia al crear, jugar y explorar se generan interdependencias entre los sistemas internos que en articulación con los estímulos del medio posibilitan un proceso creativo de co-construcción que Ilamamos aprendizaje, por tanto, "la arquitectura cerebral nunca se haIla separada de otros sistemas... [siendo] un modelo integrado y no compartimentado [en el que] la emoción interfiere la razón y la razón modifica la emoción" (Hoyuelos y Riera, 2015, pp. 39-40).

En síntesis, este breve recorrido plantea rupturas en la forma de concebir el aprendizaje desde una perspectiva lineal, por lo que resulta pertinente plantear otras formas de comprender que "el aprendizaje no es un amontonamiento sucesivo de cosas que se van reuniendo, sino que se trata de una red o trama de interacciones neuronales muy complejas y dinámicas, que van creando estados generales cualitativamente nuevos en el cerebro humano" (Assmann, 2002, p. 39). Por tanto, al trasladar este principio a la comprensión del aprendizaje en la infancia se logra percibir que los niños poseen una capacidad natural para aprender.

\section{Resignificación del aprendizaje: la infancia aprendiente}

Considerar la vida como un proceso de constante aprendizaje lleva a reconocer que el ser humano es aprendiente por naturaleza y que las adversidades que se gestan en el acto de aprender son creadas por las dinámicas relacionales humanas y no por la falta de capacidades. Entrar en contacto con esta premisa tensiona las concepciones que imperan en las relaciones educativas y en sí sobre el estudio del aprendizaje en la infancia en toda su dimensión. Estas ideas son apoyadas por Tonucci (2002), 
Maturana (2002) y García (2014), quienes a partir de sus estilos particulares invitan a resignificar a los niños desde las posibilidades que se sitúan en el ahora, pues estas se interrelacionan con las transformaciones estructurales que tienen lugar en las experiencias dadas en coexistencia con el mundo. Al respecto, Bateson (1998) indica que "la palabra 'aprendizaje' indudablemente denota un cambio de alguna clase. Decir qué clase de cambio, es un asunto delicado" (p. 199). Por tanto, este planteamiento permite reflexionar sobre la importancia de reconocer y abrir nuevos caminos que posibiliten comprender que el accionar en el mundo y en sí la experiencia perceptiva en él, genera movilizaciones que pueden considerarse aprendizajes.

Esta postura ratifica el valor de la infancia por sí misma y estimula una mirada que trasciende la visión fragmentada y reduccionista del aprendizaje. Asimismo, invita a la educación a volver a las raíces de lo humano y recobrar aquella sensibilidad infantil que permite ver el mundo desde una óptica distinta, que resignifica la construcción natural de conocimientos a partir de las emergencias que se configuran en interdependencia con los sistemas perceptivos, comunicativos, emocionales y de movimiento que actúan como detonantes de su aprendizaje. En este marco, se acude a la enacción como principio de la autoorganización, pues esta coloca en funcionamiento un proceso cognitivo global que está inmerso en la mente, asociada a la cognición corporizada, desde un intercambio del entorno biológico, social y cultural que posibilita un conocimiento en el que "el mundo y quien lo percibe, se definen recíprocamente" (Varela, Thompson y Rosch, 1997, p. 202).

Conforme a lo dicho, se puede afirmar que la acción cognitiva del niño es un proceso en potencia, que se transforma de una condición constante de aprendizaje a una situación total de aprendiencia, aspecto definido por Assmann como: "[...] "estar-en-proceso-de-aprender" [...] acto existencial que caracteriza [...] la dinámica de los seres vivos" (2002, p. 15). En este sentido, la experiencia del niño en relación con el aprendizaje inicia desde la movilización de las estructuras cognitivas y emocionales que emergen en las interacciones sociales, culturales, familiares y educativas que configuran al niño.

En este orden de ideas, el juego en la infancia como una actividad que teje las relaciones sociales, afectivas y culturales, inmersas en las experiencias vividas en el entorno natural, resignifica la realidad y dota de sentido los aprendizajes construidos. Aspecto que se asocia con la recursividad y el funcionamiento en red que poseen los organismos vivos y que lleva a comprender que existe circularidad en las interacciones sensomotrices y patrones organizados emergentes, a partir de relaciones con el entorno, que reconocen la existencia de otras experiencias corporales y de percepción. Es así, como "la cognición depende de las experiencias originadas en la posesión de un cuerpo con diversas aptitudes sensorio-motrices [...] estas aptitudes [...] están encastradas en un contexto biológico, psicológico y cultural más amplio" (Varela, Thompson y Rosch, 1997, p. 203).

En definitiva, hacer un recorrido por algunas hueIlas del aprendizaje resignifica a la infancia y su papel en el aprender desde una perspectiva activa, recursiva, reconfigurativa y en continua transformación. De ahí que resulte imperativo reconocer la existencia de los múltiples factores expresados en este texto, asociados al proceso natural que sitúa al niño como sujeto aprendiente, autoorganizado y creativo, para que de esta manera se entre en una actitud de búsqueda constante de experiencias que conduzcan a un ejercicio real y significativo para la infancia.

\section{Referencias}

Assmann, H. (2002). Placer y ternura en la educación. Madrid: Narcea.

Alzate, M. (2003). La infancia: concepciones y perspectivas. Pereira: Papiro.

Bateson, G. (1998). Pasos hacia una ecología de la mente. Buenos Aires: Lohlé, Planeta.

Capra, F. (1996). La trama de la vida. Una nueva perspectiva de los sistemas vivos. Barcelona: Anagrama.

De Corte, E. (2016). Avances históricos en el entendimiento del aprendizaje. En La naturaleza del aprendizaje. Usando la investigación para inspirar la práctica (pp. 34-55). Panamá: OCDE, 
OIE - Unesco, Unicef Lacro. Recuperado de http://panorama.oei.org.ar/_dev/wp-content/ uploads/2017/09/UNICEF_UNESCO_OECD_ Naturaleza_Aprendizaje_.pdf

García, M. R. (2014). Infancia y modernidad: José Martí y "La Exposición de París". Política y Cultura, 41, 191-205. Recuperado de http://www. scielo.org.mx/scielo.php?script=sci_arttext\&pi$\mathrm{d}=$ S0188-77422014000100009

Hoyuelos, A. y Riera, M. (2015). Complejidad y relaciones en educación infantil. Barcelona: Octaedro.

Martínez, M., Pérez, M. y Sierra, B. (2014). Incidencia de los hábitos educativos en el aprendizaje del alumnado de educación infantil. Enseñanza \& Teaching, 32(2), 147-175. https:// doi.org/10.14201/et2014321147176

Maturana, H. (2002). Amor y juego: fundamentos olvidados de lo humano. Desde el patriarcado a la democracia. Chile: Comunicaciones Noreste.
Pérez, K. y Álvarez, J. (2016). Procesos psicológicos de enseñanza y de aprendizaje. Motivación académica a partir de un programa de responsabilidad social en niños de primaria mayor. Revista de Investigación Educativa, 3, 61-67.

Portellano, J. A. (2008). Neuropsicología infantil. Madrid: Síntesis.

Ruiz, G. (2013). La teoría de la experiencia de John Dewey: significación histórica y vigencia en el debate teórico contemporáneo. Foro de Educación, 11(15), 103-124. https://doi. org/10.14516/fde.2013.011.015.005

Salas, R. E. (2012). Estilos de aprendizaje a la luz de la neurociencia. Bogotá: Magisterio.

Sánchez, L. y Andrade, R. (2014). Inteligencias múltiples y estilos de aprendizaje, diagnóstico y estrategias para su potencialización. México D. F.: Alfaomega, Instituto de Ciencias de la Educación Estado de México.

Tonucci, F. (2002). Entrevista. Barbecho: Revista de Reflexión Socioeducativa, 1, 4-7. Recuperado de http://www.barbecho.uma.es/DocumentosPDF/BARBECHO1/A1B1.PDF

Varela, F. (1998). Conocer: las ciencias cognitivas: tendencias y perspectivas. Cartografía de las ideas actuales. Barcelona: Gedisa.

Varela, F. Thompson, E. y Rosch, E. (1997). De cuerpo presente. Las ciencias cognitivas y la experiencia humana. Barcelona: Gedisa. 